\title{
A Global Game with Strategic Substitutes and Complements: Comment
}

\author{
Eric Hoffmann*, Tarun Sabarwal** \\ University of Kansas \\ 1460 Jayhawk Blvd. \\ Lawrence, KS 66045
}

September 15, 2014

\begin{abstract}
In a 2007 paper, "A global game with strategic substitutes and complements", by Karp, L., I.H. Lee, and R. Mason, Games and Economic Behavior, 60(1), 155-175, an argument is made to show existence of Bayesian-Nash equilibrim in global games that may include both strategic substitutes and complements. This note documents a gap in the proof of that statement.
\end{abstract}

\section{Introduction}

The global games method (as proposed by Carlsson and van Damme (1993)) shows that in some classes of games with strategic complements, the issue of multiple equilibria can be resolved with a slight relaxation of common knowledge about an underlying fundamental. Recall that games with strategic complements formalize the idea that observing a higher action from an opponent makes it more beneficial for a given player to also choose a higher action. In these cases, multiple equilibria arise easily. Carlsson and van Damme (1993) work with a 2-player, 2-action coordination game. Morris and Shin (2003) extend this to the case of infinitely many players. Both show that when players observe private, noisy signals about a fundamental, then iterated deletion of strictly dominant strategies produces an "essentially unique" Bayesian-Nash equilibrium strategy, which is monotone in the signal.

Karp, Lee, and Mason (2007) (KLM from here on) investigate a model similar to the infinite player game in Morris and Shin (2003), but allow for congestion effects. This introduces varying degrees of strategic substitutes in the model. Recall that games with strategic substitutes formalize the idea that observing a higher action from an opponent makes it more beneficial for a given player to choose a lower action. In general, games with strategic substitutes are harder to work with. In particular, a finite-player game with strategic substitutes does not necessarily have a pure-strategy Nash equilibrium (confer Roy and Sabarwal (2012)). Moreover, in a finite-player game with both strategic complements and substitutes, pure-strategy Nash equilibrium does not necessarily exist (confer Monaco and Sabarwal (2014)).

\footnotetext{
${ }^{*}$ Corresponding Author. Department of Economics, University of Kansas. Email: eric3353@ku.edu

** Department of Economics, University of Kansas, Email: sabarwal@ku.edu
} 
KLM consider a specialized version of games that may have both strategic complements and substitutes and focus on symmetric Bayesian-Nash equilibrium. One of their results (Proposition 1, page 161 in their paper) is that in the Bayesian game associated with the underlying complete information game, a Bayesian-Nash equilibrium always exists. In this note, we document a gap in their proof of this statement.

\section{Model and Assumptions}

The model in KLM is as follows: There is a continuum of agents of mass 1, indexed $i \in[0,1]$, each considering an action " 1 " or " 0 ". There is a payoff-relevant state given by $\theta \in \mathbb{R}$. Each player $i$ observes a private signal of the state $x_{i}=\theta+\eta_{i}$, where $\eta_{i}$ is a random variable drawn uniformly from $[-\varepsilon, \varepsilon], \varepsilon>0$, and then chooses an action. KLM define the strategy space as the set of all distributional strategies $y_{i}$ so that for any signal $x \in \mathbb{R}, y_{i}(x)$ gives the probability of choosing action 1. The strategy space for a player is denoted $Y$ and is endowed with the sup-norm metric: for any $y, y^{\prime} \in Y$,

$$
d\left(y, y^{\prime}\right)=\sup _{x \in \mathbb{R}}\left|y(x)-y^{\prime}(x)\right|
$$

KLM assume that for a given player $i$, the actions of other players affect player $i$ only through the proportion of other players that play 1 . This proportion is denoted $\alpha \in[0,1]$. In the complete information setting, utility depends on the underlying state $\theta$ and on $\alpha$ as follows

$$
U_{i}(\theta, \alpha)=\theta+f(\alpha)
$$

where $f:[0,1] \rightarrow \mathbb{R}$ is an interaction function which captures the congestion effect. The function $f$ is assumed to be an analytic function. When $f$ is everywhere increasing, the game is one of strategic complements. When $f$ is decreasing, we have strategic substitutes. When $f$ is neither always increasing nor always decreasing, we have both strategic complements and substitutes present, depending on the slope of $f$.

With incomplete information, KLM restrict attention to symmetric equilibrium. In particular, if each of player $i$ 's opponents plays the same strategy $y$, then the proportion of individuals choosing action 1 given realization of state $\theta$ is

$$
\alpha(\theta, y)=\int_{\theta-\varepsilon}^{\theta+\varepsilon} y(z) d z
$$

When player $i$ observes signal $x$, expected utility of player $i$ from playing $y_{i}$ given others are 
playing $y$ is

$$
u_{i}\left(x, y_{i}, y\right)=\left(\frac{1}{2 \varepsilon} \int_{x-\varepsilon}^{x+\varepsilon}(\theta+f(\alpha(\theta, y))) d \theta\right) y_{i}(x)
$$

In this setting, KLM state the following proposition.

Proposition 1. (KLM, page 161) In the game of incomplete information, there is an equilibrium in distributional strategies.

KLM provide a proof of Proposition 1 on page 168 in their paper, invoking Glicksberg's fixed point theorem (Glicksberg, 1952). Glicksberg's theorem requires compact strategy spaces. KLM use the following paragraph to justify that the strategy space is compact in the sup-norm topology:

From Milgrom and Weber (1985, Theorem 1), each player's set of distributional strategies is a compact, convex metric space in the weak topology. In order to establish continuity of expected payoffs, we shall use the sup-norm metric on the set of distributional strategies. The topology induced by this metric is necessarily stronger than the weak topology, since the weak topology ensures continuity only of linear functionals. Hence each player's set of distributional strategies remains compact under this metric.

The last sentence, starting with "Hence each player's...", is not justified by the previous statements. Certainly, a topology stronger than the weak topology may ensure continuity of functions other than just linear functionals. But that means more open sets, and hence more open covers to test for compactness. Therefore, compactness is harder to satisfy under stronger topologies. We present a class of counter-examples to the claim that the set of strategies used in KLM is compact in the sup-norm topology.

Claim 1. The set $Y$ of player $i$ 's strategies is not compact in the sup-norm metric.

Proof. Suppose to the contrary that $Y$ is compact in the sup-norm metric. Fix $\varepsilon \in\left(0, \frac{1}{2}\right)$ arbitrarily, and consider the open cover of $Y$ given $\{B(y, \epsilon): y \in Y\}$, where each $B(y, \epsilon)$ is the open ball of radius $\epsilon$ according to the sup-norm metric. Let $\left\{B\left(y^{n}, \epsilon\right): n=1, \ldots N\right\}$ be a finite sub-cover. Fix $\left\{x_{n}\right\}_{n=1}^{N}$ to be an arbitrary collection of points in $\mathbb{R}$, and consider any strategy $z_{i} \in Y$ that satisfies

$$
z_{i}\left(x_{n}\right) \in \begin{cases}{\left[0, y_{i}^{n}\left(x_{n}\right)-\varepsilon\right)} & \text { if } y_{i}^{n}\left(x_{n}\right)>\varepsilon \\ \left(y_{i}^{n}\left(x_{n}\right)+\varepsilon, 1\right] & \text { if } y_{i}^{n}\left(x_{n}\right) \leqq \varepsilon\end{cases}
$$

Then for each $n$, either $z_{i}\left(x_{n}\right)>y_{i}^{n}\left(x_{n}\right)+\varepsilon$ or $z_{i}\left(x_{n}\right)<y_{i}^{n}\left(x_{n}\right)-\varepsilon$, so that $\left|z_{i}\left(x_{n}\right)-y_{i}^{n}\left(x_{n}\right)\right| \geq$ $\varepsilon$. Therefore, for each $n$,

$$
d\left(z_{i}, y_{i}^{n}\right)=\sup _{x \in \mathbb{R}}\left|z_{i}(x)-y_{i}^{n}(x)\right| \geq\left|z_{i}\left(x_{n}\right)-y_{i}^{n}\left(x_{n}\right)\right| \geq \varepsilon
$$


so that for each $n, z_{i} \notin B\left(y_{i}^{n}, \varepsilon\right)$. This implies that $z_{i} \notin \cup_{n=1}^{N} B\left(y_{i}^{n}, \varepsilon\right)$, a contradiction to the fact that $\left\{B\left(y^{n}, \epsilon\right): n=1, \ldots N\right\}$ is a finite sub-cover. Consequently, $Y$ is not compact in the sup-norm metric.

In the absence of compactness, Glicksberg's fixed point theorem cannot be applied.

\section{References}

[1] Carlsson, H., \& Van Damme, E. (1993). Global games and equilibrium selection. Econometrica, 989-1018.

[2] Glicksberg, I. L. (1952). A further generalization of the Kakutani fixed point theorem, with application to Nash equilibrium points. Proceedings of the American Mathematical Society, 3(1), 170-174.

[3] Karp, L., Lee, I. H., \& Mason, R. (2007). A global game with strategic substitutes and complements. Games and Economic Behavior, 60(1), 155-175.

[4] Milgrom, P. R., \& Weber, R. J. (1985). Distributional strategies for games with incomplete information. Mathematics of Operations Research, 10(4), 619-632.

[5] Monaco, A. J., Sabarwal, T., (2014). Games with Strategic Heterogeneity (No 201240, Working Papers Series in Theoretical and Applied Economics). University of Kansas, Department of Economics.

[6] Morris, S., Shin, H.S. (2003). Global games: Theory and applications. In: Dewatripont, M., Hansen, L., Turnovsky, S. (Eds.), Advances in Economics and Econometrics. Proceedings of the Eighth World Congress of the Econometric Society. Cambridge Univ. Press, Cambridge.

[7] Roy, S., \& Sabarwal, T. (2012). Characterizing stability properties in games with strategic substitutes. Games and Economic Behavior, 75(1), 337-353. 